\title{
INFLUÊNCIA DO TAMANHO DA AMOSTRA NA ESTIMATIVA DA RIQUEZA EM ESPÉCIES EM LEVANTAMENTOS DE ABELHAS SILVESTRES (HYMENOPTERA, APOIDEA)
}

\author{
José Ricardo Cure ${ }^{1}$ \\ Guilherme S. Bastos Fo. ${ }^{2}$ \\ Ma. José Faccini de Oliveira ${ }^{2}$ \\ Og Francisco de Souza ${ }^{3}$
}

\begin{abstract}
The effect of area size, number of collecting hours and months surveyed, on the estimation of relative richness of bees is analized. In order to characterize a biogeographical region, it is suggested to collect various samples of no less than 400 especimens for each one, in contrasting localities within a region, utilizing the usual standardized methodology for wild bees collecting. Thus, it is possible to have a better profit of the effort invested in the survey, making it possible to estimate the relative species richness among different biogeographical regions, in shorter periods of time.
\end{abstract}

\section{INTRODUÇÃO}

A metodologia básica para levantamentos padronizados de abelhas foi estabelecida e discutida, principalmente, nos trabalhos de Sakagami et alii (1967) e de Laroca (1974). Esta é apropriada para amostrar insetos móveis, como as abelhas associadas a um recurso específico, geralmente conspícuo, como são as flores. Devido ao fato de que é normalmente necessária a captura individual, sobre as flores, com rede entomológica, o esforço dispendido em levantamentos de abelhas é muito grande.

Além do anotado, para se ter uma idéia sobre a riqueza relativa em espécies de vastas regiōes geográficas, é necessário o levantamento de várias áreas dentro da mesma região. Como é sabido de levantamentos de abelhas já realizados, as espécies predominantes e sua abundância relativa variam de área para área dentro da mesma região biogeográfica.

Assim, para mostrar vastas regiões, em períodos de tempo razoáveis, torna-se neœssário um melhor aproveitamento do esforço investido.

Conhecer o efeito causado pela redução do tamanho da área, do número de horas por coleta e dos meses amostrados, é um dos objetivos deste trabalho. Essas informações, permitirão melhor planejamento das coletas. Um resultado desejável seria o de fixar o tamanho mínimo que deverá ter a amostra de uma área, de forma a ser representativa da riqueza relativa de espécies naquela área quando comparada com outra. Dessa forma, poderemos amostrar vastas regiões geográficas num intervalo de tempo menor.

1. Museu de Entomologia. Departamento de Biologia Animal. Universidade Federal de Viçosa. 36570 , Viçosa-MG - Bolsista do CNPq.

2. Estudante de Agronomia. Universidade Federal de Viçosa.

3. Estudante doutorado Dep. Pure \& Aplied Biology/Imp. College. 


\section{MATERIAL E MÉTODOS}

Durante um ano, de forma padronizada, coletaram-se intensivamente ( 8 horas por semana) abelhas silvestres, sem escolha, com rede entomológica, num campo de pastagem em sucessão ecológica em Viçosa, MG. Uma descrição detalhada das sub-áreas e de outras questões relacionadas com o clima, melisofauna e composição florística, poderá ser consultada em outro trabalho (Cure et alii, em preparação). O levantamento foi realizado entre março de 1988 e março de 1989.

A área de amostragem é uma encosta de aproximadamente 2 ha que foi dividida em 4 sub-áreas (Z1, Z2, Z3, Z4), cada uma delas amostrada 2 horas/semana. As subáreas Z2 e Z4 representaram as partes mais baixas da encosta. A seqüência de amostragem nas sub-áreas foi sorteada em cada coleta. O número total de espécies coletado foi de 176 e o número total de indivíduos de 1889 (45\% destes, pertencentes a espécies eusociais).

As diferentes análises foram comparadas com os dados de um levantamento realizado por Cure (1983) em Curitiba, PR, Brasil, com metodologia idêntica e tamanho de área semelhante. Vale ressaltar que esses dados referem-se a uma área com menor diversidade de abelhas que a encontrada em outros levantamentos realizados nas vizinhanças de Curitiba (Laroca, 1974; Sakagami \& Laroca, 1971; Sakagami et alii, 1967; Bortoli, 1987). Isto, por se tratar de um local bastante influenciado pelo processo de urbanização. O número de espécies coletado nesse levantamento foi de 123 e o número de indivíduos 1449 (3\% deles correspondentes a espécies eusociais). Esses dados serão referidos como PCIDADE.

Quatro conjuntos de dados foram utilizados, com os seguintes objetivos:

1) Comparação entre diferentes tamanhos da área. Os dados utilizados foram os resultados de $\mathrm{Z} 1$, de $\mathrm{Z} 1+\mathrm{Z} 2$, de $\mathrm{Z} 1+\mathrm{Z} 2+\mathrm{Z} 3$ e de $\mathrm{Z} 1+\mathrm{Z} 2+\mathrm{Z} 3+\mathrm{Z} 4$.

2) Comparação entre diferentes esforços de coleta. Neste caso obtivemos dois conjuntos de dados, cada um deles representando aproximadamente a metade do esforço de coleta ( 4 horas cada um), independentemente das áreas. No primeiro conjunto de dados (ESFORÇO 1), foram eliminadas, de forma alternada, as primeiras 4 ou as últimas 4 horas de coleta, de forma a incluir nesses dados o intervalo total de horário amostrado. $\mathrm{O}$ segundo conjunto de dados (ESFORÇO 2), foi o complemento do primeiro.

3) Comparação dos resultados sazonais, independentemente das áreas. Para isto as coletas dividiram-se em três períodos: março-junho (MAR-JUN), julho-outubro (JULOUT) e novembro-março (NOV-MAR).

4) Comparação dos resultados totais (TOTAL) obtidos em Viçosa, com um subconjunto de dados resultante de um esforço de coleta equivalente à escolha de duas subáreas (uma alta e uma baixa - por sorteio Z1 e Z4), no período de maior abundância de abelhas (NOV-MAR) e com o número de horas de coleta diária reduzidos, como indicado no item 2 (no caso, por sorteio, foi escolhido o ESFORÇO1). Os dados resultantes desta combinação serão chamados de PARCIAL.

Em cada análise foi comparado o número de espécies efetivamente coletado no campo, na menor das amostras, com os valores calculados a partir dos totais das outras amostras. Os valores esperados estão na Tabela 2 , onde também se encontram, marcados com um asterisco, os valores efetivamente encontrados no campo.

Para calcular o número de espécies, caso o esforço de coleta fosse menor, utilizouse o método de Sanders (1968), com a correção recomendada por Hulbert (1971). Esta 
Vol. 7(1-2), 1990

correção elimina os problemas anotados por Fager (1972) de superestimação do número esperado de espécies. Na fórmula de Hulbert é possível calcular o número esperado de espécies numa sub-amostra com $n$ indivíduos colhidos ao acaso entre uma coleção total de $N$ indivíduos e $S$ espécies. Plotando esses resultados, de forma similar à proposta por Sanders, pode-se estimar a diversidade relativa entre diferentes amostragens, pela comparação entre as curvas resultantes. Os dados básicos para todas as análises estão representados na Tabela 4.

\begin{tabular}{llll}
\hline & $z 1$ & $z 2$ & $z 3$ \\
\hline$z 2$ & 54 & & \\
$z 3$ & 57 & 57 & \\
$z 4$ & 53 & 59 & 53 \\
\hline
\end{tabular}

\begin{tabular}{cccc}
\hline & $z 3+z 4$ & $z 2+z 4$ & $z 2+z 3$ \\
\hline$z 1+z 2$ & 66 & & \\
$z 1+z 3$ & - & 60 & \\
$z 1+z 4$ & - & - & 63 \\
\hline
\end{tabular}

\begin{tabular}{ll}
\hline & $z 3$ \\
\hline$z 1+z 2$ & 60 \\
$z 1+z 2+z 3$ & 78 \\
$z 1+z 2+z 3+z 4$ & 73 \\
\hline
\end{tabular}

Tabela 1 Similaridade entre as sub-areas (Indice de Sorensen ew $x$ ). As sub-areas $\mathrm{Z} 2$ e $Z 4$ s $\mathbf{z}$. as mais baixas da drea amostrada.

\section{RESULTADOS E DISCUSSÃO}

1. análise

A importância das amostras em cada uma das sub-áreas em número de indivíduos e de espécies foi a seguinte:

indivíduos $\mathrm{Z} 1$ (539) $>\mathrm{Z3}(481)>\mathrm{Z} 4(432)>\mathrm{Z2}(422)$

espécies $\mathrm{Z} 3(99)>\mathrm{Z} 1(89)>\mathrm{Z} 2(79)>\mathrm{Z} 4$ (77)

A subárea Z3, com $26 \%$ do total de indivíduos, teve a maior riqueza em espécies, $57 \%$ do total, enquanto Z4, a sub-área mais pobre em espécies, teve $23 \%$ dos indivíduos, representados por $44 \%$ das espécies.

A similaridade entre as diferentes sub-áreas (Tabela 1), consideradas individualmente, ficou entre $53 \%$ e $59 \%$, entretanto, somadas duas a duas, isto é, aumentando o tamanho da área, a similaridade oscilou entre $60 \%$ e $68 \%$. Quando comparamos Z3 com diferentes tamanhos de área $(\mathrm{Z} 1, \mathrm{Z} 1+\mathrm{Z} 2, \mathrm{Z} 1+\mathrm{Z} 2+\mathrm{Z} 3, \mathrm{Z} 1+\mathrm{Z} 2+\mathrm{Z} 3+\mathrm{Z} 4)$ a similaridade aumenta com o aumento da área. $\mathrm{O}$ único caso em que o padrão foi revertido é quando comparamos $\mathrm{Z} 3 \operatorname{com} \mathrm{Z} 1+\mathrm{Z} 2+\mathrm{Z} 3+\mathrm{Z} 4$. Isto porque a menor similaridade existente foi entre $\mathrm{Z} 3$ e $\mathrm{Z} 4$.

$\mathrm{O}$ menor número de espécies exclusivas encontrou-se em Z2 e Z4 (16 espécies em cada uma), e os maiores em Z1 e Z3 ( áreas mais baixas de encosta, Z2 e Z4, apresentaram a maior similaridade (59\%).

Na Tabela 2 observa-se que para diferentes combinações das sub-áreas, em subamostras de 481 indivíduos, teríamos coletado os seguintes números de espécies (com asterisco o valor efetivamente obtido no campo):

$$
\begin{aligned}
& \mathrm{Z} 3(101 *)>\mathrm{Z} 1+\mathrm{Z} 2+\mathrm{Z} 3(96)>\mathrm{Z} 1+\mathrm{Z} 2+\mathrm{Z} 3+\mathrm{Z} 4(95)> \\
& >\mathrm{Z} 1+\mathrm{Z} 2(89)>\mathrm{Z} 1(84)>\mathrm{PCIDADE}(76)
\end{aligned}
$$


De acordo com isto: a - se tivéssemos amostrado apenas $\mathrm{Z} 3$, a estimativa do número de espécies seria ainda maior que coletando nas quatro sub-áreas, b - não existiria dferença quantitativa (número de espécies) acrescentando $Z 4$ ao conjunto $Z 1+Z 2+Z 3$, c - amostrando apenas Z1 teríamos tido uma estimativa muito inferior do que amostrando $\mathrm{Z} 3$, d - em qualquer um dos casos a estimativa do número de espécies seria bem superior à encontrada em PCIDADE.

$\mathrm{O}$ fato da maior similaridade entre sub-áreas aparecer entre aquelas mais baixas, ou entre aquelas mais altas, é um indicativo de que, incluindo heterogeneidade na amostragem, é possível obter maior diversidade do que aumentando apenas a área de amostragem numa região mais homogênea. Quando reduzimos à metade o tamanho da área, ainda podemos calcular de forma adequada a riqueza relativa de espécies do local.

Comparando as curvas de riqueza em espécies entre as diferentes amostras (Figura 1), observa-se que para um valor de aproximadamente 400 individuos, as diferenças entre elas já são bem contrastantes.

\section{2. análise}

Na Figura 2 são representados os valores obtidos com diferentes esforços de coleta (Tabela 2). Constata-se que não existe quase nenhuma diferença entre os resultados de ESFORÇO1, ESFORÇO2 e TOTAL. Para uma sub-amostra de 901 indivíduos, os resultados esperados para o número de espécies seriam (com asterisco o valor efetivamente obtido no campo):

\section{TOTAL $=\operatorname{ESFORÇO2~(129)~}>$ ESFORÇO1 $\left(126^{*}\right)>$ PCIDADE $(100)$}

Existe pouca diferença entre a sub-amostra de TOTAL e as de ESFORÇOl e ESFORÇO2, mas todas contrastam com PCIDADE. A diminuição do esforço de 8 para 4 horas modificou pouco os resultados. Isto sugere que, utilizar essas horas a mais, numa outra área, teria sido mais apropriado, do ponto de vista da estimativa da riqueza relativa de espécies da região.

Comparando as curvas da Figura 2 observa-se, como na análise anterior, que para amostras de 400 indivíduos, as diferenças entre elas já são bem contrastantes.

\section{Análise}

Na Figura 3, os resultados NOV-MAR fornecem a melhor estimativa do total de espécies. Para uma sub-amostra com 388 indivíduos, a estimativa do núniero de espécies nos diferentes períodos seria (com asterisco o valor efetivamente obtido no campo):

TOTAL (87) > NOV-MAR (86) > MAR-JUN (77*) > PCIDADE (69) > JUL-OUT (51)

A estimativa para o número de espécies, praticamente não difere entre TOTAL e o intervalo NOV-MAR. No contraste com PCIDADE, mesmo o intervalo MAR-JUN, com pouca abundância de abelhas, fornece uma indicação de diversidade maior do que PCIDADE. Todavia, a estimativa do número total de espécies é bastante modificada, quando as coletas são restritas a um período de meses (JUL-OUT) com pouca abundância de indivíduos. 
Nimero de individuos

\begin{tabular}{|c|c|c|c|c|c|c|c|c|c|c|c|c|c|c|c|c|c|}
\hline Anostras & 10 & so & 150 & 276 & 388 & 400 & 481 & 529 & 539 & 901 & 961 & 987 & 988 & 1442 & 1449 & 1874 & 1889 \\
\hline
\end{tabular}

\begin{tabular}{|c|c|c|c|c|c|c|c|}
\hline $\mathbf{z 1}$ & 7.1 & 23.3 & 46.5 & 77.4 & 83. 9 & 88.0x & \\
\hline $\mathbf{z} 3$ & 8. 1 & 27.3 & 54.5 & 92.2 & $101.0=$ & & \\
\hline $21+2 z$ & 7.3 & 23.6 & 47.0 & 80.9 & 88. 9 & 94.0119 .6 & $123.0 \%$ \\
\hline $21+22+23$ & 7.6 & 25.0 & 50.3 & 87. 1 & 95.7 & 101.3129 .0 & \\
\hline$z 1+z 2+z 3+z 4$ & 7.6 & 24.9 & 50.1 & 86. 7 & 95.2 & 100.8128 .3 & \\
\hline
\end{tabular}

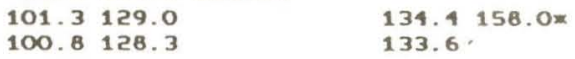

\begin{tabular}{|c|c|c|c|c|c|c|c|c|}
\hline \multirow{2}{*}{$\begin{array}{l}\text { MAR-JUN } \\
\text { JUL-OUT }\end{array}$} & 7.4 & 23.1 & 46.1 & \multicolumn{2}{|l|}{$77.0 \%$} & & & \\
\hline & 5.5 & 16. 4 & 31.9 & 51.0 & & 58. O\% & & \\
\hline NOV-MAR & 8.1 & 26.1 & 51.4 & 86. 0 & 87.4 & 100.5 & 128.1 & $133.0 \mathrm{~m}$ \\
\hline ESFORCO1 & 7.7 & 25.1 & 50.6 & & 86. 2 & & $126.0 \%$ & \\
\hline ESFORCOZ & 7.4 & 24.7 & 50.1 & & 87.2 & & 129.6 & $135.0 \times$ \\
\hline
\end{tabular}

PARCIAL 8.2 $26.452 .0 \quad 71.0 \%$

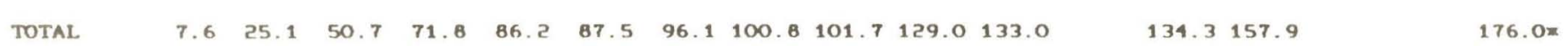

\begin{tabular}{llllllllll}
\hline PCIDADE & 7.9 & 24.8 & 44.1 & 58.7 & 68.6 & 69.6 & 75.7 & 100.1 & $123.0=$
\end{tabular}

Tabela 2. Nimero de especies coletadas nas aiferentes amostras (marcado com e mimero de especies estimado pelo wetodo de Sanders (1968), conforme wodificacko de Furlbert (1971). para diferentes tamanhos de amostra. 
Como a nossa intenção é coletar o maior número relativo de espécies possivel num curto intervalo de tempo, convém escolher como época de coleta aquela com maior abundância de abelhas. Neste exemplo, seria o intervalo NOV-MAR.

\begin{tabular}{lccccc}
\hline Amo stras & H & Hmax & J & $\alpha$ & Xind. \\
\hline$Z 1$ & 1.38 & 1.94 & .71 & 29.87 & 41 \\
$Z 3$ & 1.59 & 2.00 & -79 & 39.00 & 50 \\
$Z 1+Z 2$ & 1.45 & 2.09 & .69 & 37.44 & 44 \\
$Z 1+Z 2+Z 3$ & 1.54 & 2.20 & .70 & 45.36 & 42 \\
$Z 1+Z 2+Z 3+Z 4$ & 1.54 & 2.24 & .69 & 47.07 & 39 \\
\hline MAR-JUN & 1.41 & 1.89 & -75 & 16.54 & 48 \\
JUL-OUT & 1.03 & 1.76 & .58 & 16.64 & 40 \\
NOV-HAR & 1.60 & 2.12 & .75 & 41.66 & 41 \\
\hline ESFORCO1 & 1.54 & 2.10 & -73 & 39.99 & 44 \\
ESFORCO2 & 1.50 & 2.13 & .70 & 42.24 & 44 \\
\hline PARCIAL & 1.52 & 1.85 & .82 & 31.00 & 45 \\
\hline TOTAI & 1.55 & 2.25 & .69 & 47.44 & 40 \\
\hline PCIDADE & 1.52 & 2.09 & .73 & 31.85 & 43 \\
\hline
\end{tabular}

\section{Tabela 3. Valores de alguns indices de diversidade para as várias amostras. ( $\mathrm{H}^{\circ}=$ Shan- non-Wiener, Hex=valor de de $H^{*}$. $J^{\circ}=$ equitabilidade, $\alpha=$ Fisher, $x$ ind. = percentagem de especies com 1 individuo).}

\section{Análise}

Combinando os três tipos de diminuição do tamanho da amostra, conforme as três primeiras análises (menor área, menor número de meses e menor número de horas por coleta), obtivemos uma amostra, representada na Figura 4 (PARCIAL) de apenas 276 indivíduos (isto representou $6,8 \%$ do total de indivíduos coletados). Para sub-amostras desse tamanho, a estimativa do número de espécies foi (com asterisco o valor efetivamente obtido no campo):

\section{TOTAL (72) $>$ PARCIAL $\left(71^{*}\right)>$ PCIDADE (59)}

Na sub-amostra calculada a partir de TOTAL, a estimativa do número de espécies é de 72, o que praticamente não difere do resultado obtido no campo (PARCIAL), mas contrasta com PCIDADE, indicando menor riqueza em espécies nesta última.

As curvas representadas na Figura 4 indicam, novamente, bom contraste entre elas, por volta dos 400 indivíduos. 
$\mathbf{z}_{1}$

23

$21+22$

$21+22+23$

1/66; 2/23; 3/14; 4/10; 5/7; 6/9; 7,8/2; 10/3;

$11 / 2,12 / 1,14 / 2 ; 16,18,20,21,22,23,24,28,32,33,36$,

$55,68,71,93,97,380 / 1$

$21 * Z 2 * 23+Z 41 / 6 e ; \quad 2 / 25 ; 3 / 17 ; 4 / 11 ; 5 / 7 ; 6 / 8,7 / 2,8 / 6 ; 9,10 / 1 ; 11$, $12,13,15 / 2 ; 16,18 / 1 ; 21 / 2 ; 22,23,24,26,27,28$, $31,37,45,55,82,90,102,105,127,495,1$

MAR-JUN $1 / 37 ; 2 / 16 ; 3 / 5 ; 4 / 4 ; 5 / 3 ; 6 / 2 ; 6 / 3 ; 9,13,16,29$, $37,43,90 / 1$

JUL-OUT 1/23; 2/11;3/5;4/4;5/2;6/3; 7/1; 8/2; 9,11, $16,19,29,70,246 / 1$

NOY-MAR. $\quad 1 / 54 ; 2 / 27 ; 3 / 13 ; 4 / 6 ; 5 / 4 ; 6 / 2 ; 7 / 1 ; 6 / 3 ; 9 / 1$; $10 / 3 ; 11 / 2 ; 13,14,15 / 1 ; 16,18,20 / 2 ; 25,26,33,52$, $72,60,96,158 / 1$

ESFORCO 1 1/55; 2/20; 3/12; 4/5; 5/7; 6/4; 7/1; 8/3; 9/2; $10,12 / 1 ; 13 / 2 ; 14 / 3 ; 20 / 1 ; 21 / 2 ; 23,36,41,49,58$, $69,214 / 1$

ESFORCO2 1/59; 2/24; $3,4 / 11 ; 5 / 3 ; 6 / 4 ; 7,8 / 1 ; 9,10 / 2$; $11,12 / 1 ; 13 / 2 ; 14 / 3 ; 15,17,22,35,46 / 1 ; 47 / 2 ; 53$, $58,281 / 1$

PARCIAL 1/32; $2 / 18 ; 3 / 5 ; 4 / 4 ; 5 / 2 ; 6 / 1 ; 7 / 2 ; 12,13,14,15$, $19,29,45 / 1$

TOTAL $\quad 1 / 70 ; 2 / 23 ; 3 / 17 ; 1 / 12 ; 5 / 7 ; 6 / 7 ; 7 / 3 ; 6 / 6 ; 9 / 2 ;$ $10 / 1 ; 11,12,13,15 / 2 ; 16,19,21,22,23 / 1 ; 24 / 2 ; 26$, $27,28,31,37,45,56,82,88,102,105,127,495 / 1$

PCIDADE $\quad 1 / 53 ; 2 / 15 ; 3 / 44 ; 4 / 4 ; 6,7 / 3 ; 8 / 1 ; 10 / 2 ; 11 / 1 ;$ $12 / 2 ; 14,17 / 1 ; 19,22,25 / 2 ; 28,30,31,42 / 1,45 / 2$; $46 / 1 ; 51 / 2 ; 55,66,67,79,88,304 / 1$

Tabela 4. Numero de individuos - Numero de especies nas diferentes amostras. 

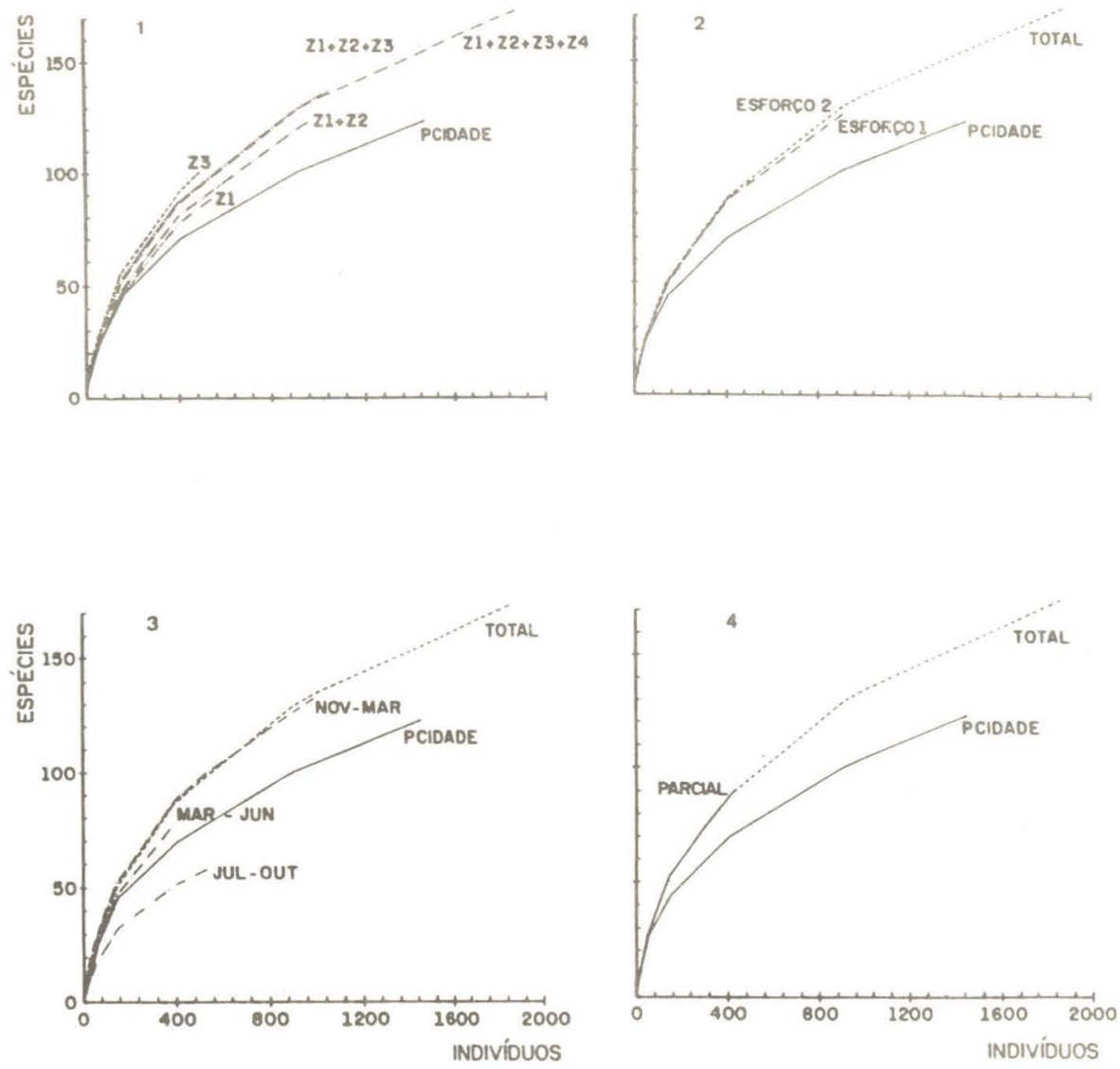

Figuras 1 a 4 - Curvas de espécies para diferentes amostras de um levantamento feito em Viçosa, MG, comparadas à de outro levantamento, PCIDADE, realizado em Curitiba, PR. 1. Efeito do tamanho da área. 2. Efeito do número de horas por coleta. 3. Efeito do período de meses amostrado. 4. Combinação da redução da área, do número de horas por coleta e do período de meses amostrado. TOTAL: total do levantamento em Viçosa. Z1, Z2, Z3, Z4 : sub-áreas do local amostrado. ESFORÇO1: diminuição à metade do número de horas por coleta. ESFORÇO2: complemento de ESFORÇO1. MAR-JUN: intervalo março a junho. JUL-OUT: intervalo julho a outubro. NOV-MAR: intervalo novembro a março. PARCIAL: efeito resultante de amostrar, com ESFORÇO 1, durante o período NOV-MAR, as sub-áreas Z1 + Z4. 


\section{CONCLUSÕES}

Na Tabela 3 são apresentados os valores de alguns índices comuns de diversidade para as amostras analisadas. Nenhum desses índices diz nada semelhante ao observado nas Figuras 1 a 4. Como foi mencionado por Hulbert (1971), duas coleções poderão ter diversidade idêntica quando calculadas pelos índices convencionais, entretanto, mostrando curvas de riqueza em espécies totalmente diferentes.

$\dot{E}$ uma constatação importante, o fato dos valores estimados para essas curvas, pelo método Sanders-Hulbert, coincidirem quase que exatamente com os realmente encontrados nas amostras (Tabela 2, valores marcados com asteriscos).

Os dados resultantes do levantamento em Viçosa, mostram que a forma das curvas de indivíduos vs espécies, nas diferentes análises propostas, varia pouco com diferentes tamanhos de área ou com diferentes esforços de amostragem (em termos de horas de coleta), porém, ela é bastante modificada dependendo dos meses amostrados.

Entre as limitações da metodologia, apontadas por Sanders (op. cit.), para as curvas de indivíduos vs. espécies, a técnica é válida somente quando os mesmos tipos de organismos são comparados, os habitats amostrados são similares (comparações dentro de habitats e não entre habitats, no sentido de McArthur, 1965, conforme citado pelo próprio Sanders) e a metodologia de amostragem é semelhante.

Outra limitação citada por esse autor, importante de ser ressaltada, é que o método pode ser utilizado para interpolar mas não para extrapolar. Isto quer dizer que a nossa proposta de diminuir o esforço de amostıagem é válida para comparações relativas mas não serve para estimar o número total de espécies presentes.

Essa limitação não interfere com a questão fundamental, que é a comparação entre as curvas provenientes de várias amostras. Parece-nos que um número de 400 indivíduos é adequado para julgar a riqueza relativa de espécies. Nas amostras analisadas, para sub-amostras de 400 indivíduos, as diferenças já foram marcantes.

Convém salientar, entretanto, que o número mínimo de 400 indivíduos deverá ser tomado com cautela, principalmente pela presença de espécies eusociais. Do ponto de vista metodológico, parece-nos oportuno sugerir que as espécies eusociais sejam amostradas simultaneamente com as outras abelhas. Não achamos conveniente eliminar da análise as espécies eusociais, precisamente porque elas interagem com as outras abelhas, e representam elementos de primeira importância na distribuição dos recursos existentes e portanto da diversidade.

A utilização de uma metodologia padronizada de coleta, em amostras com 400 indivíduos, deverá permitir uma boa representatividade das espécies solitárias. Resta, no entanto, mencionar que a proporção destas espécies em relação às sociais, é variável em diferentes regiões biogeográficas, ou mesmo em diferentes localidades da mesma região. Esta questão mereœ ainda um estudo particular. Por enquanto, devemos apenas deixar constância sobre a proporção de espécies eusociais nos levantamentos.

$\mathrm{Na}$ amostra de Viçosa, as espécies eusociais representaram 50\% do total, enquanto que em PCIDADE foi de apenas 3\%. Apesar dessa diferença, a riqueza em espécies foi bem maior em Viçosa, para a maior parte das análises.

Outra questão, já levantada por Hurlbert (op. cit.), é a possibilidade de cruzamento entre duas curvas de espécies. Isto aconteceu, na Figura 3, entre TOTAL e NOVMAR. Situações como essa, levaram Hurlbert (op. cit.) a sugerir que comparações de um único ponto da curva têm pouco significado, recomendando o exame da curva completa. 
A interpretação que esse autor deu para o cruzamento entre as curvas é que uma das amostras (no nosso caso, NOV-MAR) teria maior riqueza em espécies com individuos de baixa mobilidade, enquanto que a outra amostra (TOTAL), teria maior riqueza em espécies com individuos de grande mobilidade.

A explicação da mobilidade pode ser mais elaborada, dependendo do grupo de organismos em estudo. Pode-se pensar em probabilidade menor de coletar as espécies móveis quando a amostra é pequena. No caso das abelhas, essa questão também está ligada à heterogeneidade provocada pelos recursos florais, que não estão uniformemente distribuidos no tempo ou no espaço.

Apesar das limitações apontadas, consideramos que para um levantamento extensivo de uma região biogeográfica, a representatividade conseguida entre várias amostras pequenas, com cerca de 400 indivíduos cada uma, incluindo heterogeneidade ambiental, sazonal e temporal (horários diferentes), são apropriadas para se fazer comparações entre vastas regiões geográficas.

\section{AGRADECIMENTOS}

Aos Professores Jesús Santiago Moure e Sebastião Laroca, da Universidade Federal do Paraná, pelas sugestões. Ao Professor Fernando Amaral da Silveira CNPq/UFV, pelas sugestões e ajuda durante as coletas. Ao CNPq pelo apoio financeiro.

\section{REFERÊNCIAS BIBLIOGRĀFICAS}

BORTOLI, C. de, 1987. Estudo biocenótico em Apoidea (Hymenoptera) de uma área restrita em São José dos Pinhais (PR, sul do Brasil), com notas comparativas. Tese de Mestrado. Departamento de Zoologia. Universidade Federal do Paraná, $142 \mathrm{pp}$.

CURE, J.R., 1983. Estudo Ecológico da Comunidade de Abelhas Silvestres (Hymenoptera, Apoidea) do Parque da Cidade, Comparado ao de outras áreas de Curitiba, Paraná. Tese de Mestrado. Departamento de Zoologia. Universidade Federal do Paraná, 100 pp.

FAGER, E.W. 1972. Diversity: a sampling study. The American Naturalist 106 (949): 293-310.

HURLBERT, S.H. 1971. The nonconcept of species diversity:A critique and alternative parameters. Ecology, 52 (4): 577-586.

LAROCA, S., 1974. Estudo Feno-Ecológico em Apoidea do Litoral e Primeiro Planal to Paranaenses. Tese de Mestrado. Departamento de Zoologia. Universidade Federal do Paraná, 61 pp.

SAKAGAMI, S.F. \& S. LAROCA, 1971. Relative abundance, phenology and flower visits of apid bees in eastern Paraná, southern Brazil (Hymenoptera, Apoidea). Kony tú 39 (3): 217-230.

SAKAGAMI, S.F., S. LAROCA \& J.S. MOURE, 1967. Wild bee biocenotics in São José dos Pinhais (PR), south Brazil. Preliminary report. J. Fac. Sci. Hokkaido Univ. Ser. VI, Zool. 16 (2): 253-291.

SANDERS, H.L. 1968. Marine Benthic Diversity: A Comparative Study. The American Naturalist $102(925): 243-281$ 